\title{
Resultados de la utilización de un software para la administración de un sistema combinado en una MYPE*
}

\section{Results of the use of a software for managing \\ a combined system in a MSE}

Recibido: 10 de octubre de 2013

Revisado: 25 de abril de 2014

Aceptado: 27 de junio de 2014

Julián Roberto Acevedo Rey**

Convenio Universidad Santo Tomás e ICONTEC

\section{RESUMEN}

El incremento sostenido en el uso de los sistemas de gestión en las organizaciones ha motivado el interés de los desarrolladores de software por crear herramientas de automatización para la administración de los sistemas de gestión. Mediante la modalidad investigativa del estudio de caso, se analizan los resultados obtenidos en la empresa Comercializadora ICER de la Costa Ltda. al utilizar un software para la administración de su sistema combinado de gestión.
Los datos sobre la utilización del software se obtienen a través de observación, percepción de los usuarios y opinión de expertos. Nuestra hipótesis es que el uso de un software genera beneficios en la administración de sistemas combinados de gestión. Proponemos una Guía de Calificación de Software que sirve para orientar a las MYPES interesadas en la compra de un software para administrar sus sistemas de gestión.

Palabras claves: administración, sistema de gestión, software, sistemas de gestión combinados.

* Trabajo de grado para optar al título de Magíster en Calidad y Gestión Integral, dirigido por Mg. John Jairo Gutierrez Ordoñez.

* Especialista en Administración y Gerencia de Sistemas de Gestión de la Calidad. Gerente Consultor de HiPERiON Consultorías y Asesorías. Correo electrónico: Julian.acevedo@hiperion.com.co 


\section{ABSTRACT}

The steady increase in the use of management systems in organizations, has motivated the interest of software developers to create automation tools for management systems. Based on research case study method, the company Comercializadora ICER de la Costa Ltda is analyzed. Particularly, the use of software to manage their combined management system is discussed, Data on the use of software is obtained through observation, perception of users and expert opinion. This data is used to verify our hypothesis: that the use of software is beneficial in the combined administration of management systems. In addition, we propose a Guide for assessing the software used, as a way to guide MSEs interested in buying software capable to manage their management systems.

Keywords: Management, management system, software, combined management systems.

\section{INTRODUCCIÓN}

El software es una herramienta que cada vez más se utiliza en la industria para facilitar, dinamizar y sistematizar un conjunto de tareas o rutinas que permitan elaborar un producto. Así mismo, la importancia que ha despertado en el mundo moderno la implementación de sistemas de gestión ha generado el interés de empresas desarrolladoras de software por incluir dentro de su portafolio programas que aseguren la mejora de los sistemas de gestión.

Dicha situación ha llevado a muchas empresas a comprar software para administrar sus sistemas de gestión. Este es el caso de la Comercializadora ICER de la Costa, cuya Gerencia decidió invertir en un software para facilitar y asegurar la implementación, así como su posterior mantenimiento, de sus sistemas de gestión de Calidad, Seguridad y Salud Ocupacional. Sin embargo, no se conocen los resultados que se presentan cuando se administra un sistema combinado a través de un software. Además, se desconoce el funcionamiento y características que debe tener el software para dar cumplimiento a los requisitos de los sistemas de gestión, teniendo en cuenta que la herramienta cuenta con una serie de rutinas, de acuerdo a su diseño y funciones.

En ese sentido, el objetivo de la presente investigación es observar lo que sucede cuando interactúa el usuario con el programa, así como evaluar la percepción que tienen los usuarios frente al software y, finalmente, comparar los resultados con la opinión que tienen expertos sobre el tema.

El marco teórico está segmentado en cuatro corrientes teóricas que dan cuerpo al proyecto. La primera corriente teórica tiene que ver con la forma como se puede hacer una adecuada administración. La segunda corriente se refiere a la tecnología y la forma como ésta ha disminuido brechas espacio-temporales y ha facilitado la comunicación a través de software y tecnología. La tercera corriente se alinea al paradigma sistémico y al término "integrado", en esta investigación se usa el término "combinado", para referirnos a la interacción de más de un sistema. Finalmente, la última corriente teórica se relaciona al método de investigación adoptado para la presente investigación: "estudio de caso", el cual explica el diseño y método para su ejecución.

Ahora bien, la presente investigación se basa en la Teoría Clásica de la Administración que expone Henry Fayol $^{1}$ y los aportes que hace con respecto a la importancia de aumentar la eficiencia de la empresa a través de la distribución y disposición de los departamentos y de sus interacciones. De allí nace el interés por la estructura y funcionamiento de la organización como

1 Henry Fayol. Ingeniero de Minas. Nació en Constantinopla en 1841 y murió en Paris en el año 1925. Fue Conocido como el padre de la Teoría Clásica de la Administración. 
aspecto vital para su administración, teniendo como línea las directrices generadas desde la parte superior de la estructura.

Por otro lado, la tecnología actual, lejos de ser una herramienta que genere incertidumbre, problemas 0 perturbe, tiene como objetivo generar seguridad y confianza gracias a su capacidad de acercar a las personas en situaciones que ni la misma generación actual podría haberse imaginado. En este sentido, Bauman expresa que un factor técnico al que le cupo una función particularmente importante fue el transporte de la información: un tipo de comunicación que requiere poco o ningún desplazamiento de cuerpos físicos. ${ }^{2}$

Dejando a un lado el marco tecnológico, vital para esta investigación, se hace necesario involucrar el contexto de la combinación de los sistemas. En este sentido, esta investigación utiliza los conceptos y aportes de la Teoría General de Sistemas de Bertalanffy, que sostiene que "el todo es el conjunto de sus partes, que se relacionan de una forma dinámica e interdependiente”. Esta investigación, también se fundamenta en los aportes de Morin ${ }^{3}$ respecto al Paradigma Sistémico, la cual trasciende en mayor medida la Teoría General de Sistemas de Bertalanffy.

Finalmente, el norteamericano Robert K. Yin en el libro Caso de Estudio de investigación ${ }^{5}$ define el estudio de caso como una estrategia de investigación que puede

2 BAUMAN, Zygmunt. La globalización. Consecuencias humanas. En: Sección de obras de sociología. Brasil: Fondo de Cultura Económica. 1999. 23 p.

3 Edgar Morin. Filósofo, Político y So ciólogo Francés, nacido en Paris el 8 de julio de 1921.

4 Ludwig von Bertalanffy. Biólogo y Filósofo austriaco, nacido en Viena el 19 de septiembre de 1901 y fallecido el 12 de junio de 1972 en Buffalo, Nueva York, Estados Unidos; autor de La Teoría General de Sistemas.

5 YIN, Robert K. Caso de estudio de investigación: diseño y métodos.3 ed. Sage Publicaciones. California.: 1984. ser usado en varias situaciones, entre las cuales se encuentran los estudios organizacionales y de administración. Por ello, para nuestra investigación y problema objeto de estudio nos basamos en este método de investigación.

El presente estudio tiene como objetivo: a) Evaluar la pertinencia y eficiencia del software a partir de la observación por parte del autor de la investigación; b) Evaluar la percepción que tienen los usuarios frente al cumplimiento de las promesas de valor del software a través de la aplicación de cuestionarios; c) Comparar los resultados de la percepción y de la observación con la opinión de expertos por medio de la aplicación de una matriz de calificación de software; d) Establecer una metodología a través de una guía práctica y sencilla que permita evaluar las características más importantes de un software que administre sistemas combinados de gestión, y e) Determinar la viabilidad y pertinencia del uso del software para administrar el sistema combinado de gestión en la Comercializadora ICER de la Costa Ltda.

Para cumplir estos objetivos se plantea la siguiente hipótesis: utilizar un software favorece el manejo de un sistema combinado de gestión.

\section{METODOLOGÍA}

El presente trabajo se basa en la metodología descrita por Yin sobre el estudio de caso. Tanto las preguntas de estudio como las especificaciones, unidades de análisis, recolección de información, y la lógica que unen los datos a las especificaciones y los criterios, se usan para interpretar los resultados ${ }^{6}$. Con el fin de consolidar los componentes, la metodología se varió partiendo de las unidades de análisis, la recolección de datos y

6 Ibid., p. 27 
definiendo la lógica que unen los datos a las especificaciones. Además, se incluyó la técnica de la triangulación de los datos, cuyo fin es asegurar la imparcialidad y objetividad de los resultados alcanzados. El principio de triangulación y convergencia es una estrategia que busca dar credibilidad a la investigación de forma interna y externa en lo que concierne a la identificación de los resultados obtenidos al momento de administrar un sistema combinado de gestión a través de un software, tal como se muestra en la figura 1: ha sido ajustado y mejorado desde su puesta en marcha para asegurar que su uso y funcionamiento cumpla con las expectativas de la Comercializadora ICER de la Costa.

Según Hugo Cerda una muestra sirve para caracterizar una reducida parte de un todo, que sirve para describir las características fundamentales de aquél. Generalmente ese "todo" corresponde a la población, universo o colectivo que se investiga. ${ }^{7}$

Figura 1. Esquema del principio de triangulación.

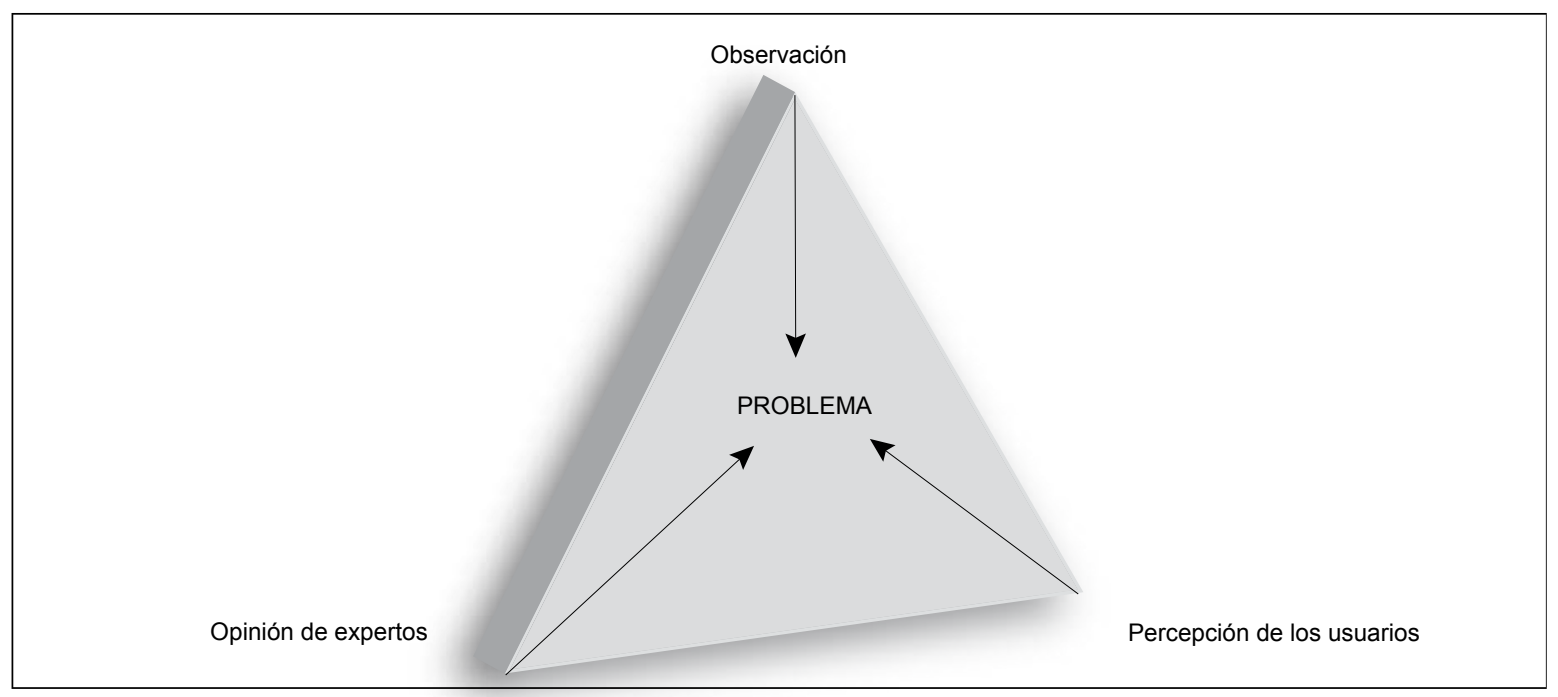

Fuente: elaboración propia.

La investigación no se limita a examinar los resultados obtenidos en cada uno de los vertices del triángulo anterior. En este sentido, uno de los objetivos de la investigación es proponer una guía que sirva en la empresa Comercializadora ICER de la Costa al momento de evaluar la utilización de un software para administrar sistemas combinados de gestión. Así mismo, la presente investigación busca que dichos resultados sean coherentes y cumplan con las expectativas planteadas en cada vertice y en el conjunto de los vertices.

Por tanto, los resultados de esta investigación buscan generar la Guía de calificación de software y no pretenden validar el software ISOSISTEM. Este software
La presente investigación analiza el caso de la empresa Comercializadora ICER de la Costa. Esta empresa cumple con las características propias de una MYPE, además se encuentra certificada con la norma técnica NTC-ISO 9001:2008 "Sistemas de gestión de la calidad. Requisitos" y con la norma técnica NTG-OHSAS 18001:2007 "Sistema de gestión en seguridad y salud ocupacional. Requisitos". Así mismo, la empresa compró un software para reducir las dificultades que ha sufrido al momento de administrar el sistema combinado de gestión.

7 CERDA. Hugo. Los elementos de la investigación. Editorial El Búho Ltda. Bogotá.2008. p. 298 
Durante la observación se realizaron 80 preguntas, a partir de seis cuestionarios, a cuatro personas con distintos cargos: Gerente, Coordinadora QHS, Líderes de proceso y Trabajadores. El objetivo de la observación y del cuestionario fue determinar la brecha existente entre las características prometidas por el software ISOSISTEM frente a su desempeño real y a la percepción de los usuarios. A partir de una matriz de calificación de software, respondida por siete expertos, se diseñó una guía de calificación de software para facilitar a las MYPES la compra de dicha herramienta.

\section{RESULTADOS Y DISCUSIÓN}

En la aplicación del método de observación, con base en las respuestas a las 80 preguntas formuladas durante los cinco momentos de observación, se estudió: a) parametrización, b) capacitación, c) puesta en marcha, d) auditoría, y e) mantenimiento, se encontraron los siguientes resultados:

- La empresa proveedora de software ofreció una revisión preliminar de la infraestructura tecnológica con la que contaba ICER Costa, lo que permitió establecer las necesidades que requería la empresa para instalar el software.

- Las competencias del Ingeniero Programador de la empresa proveedora de software permitieron y facilitaron la parametrización. Sus conocimientos se consideraron las mayores fortalezas para alcanzar los resultados deseados.

- Así mismo, las constantes pruebas realizadas a las parametrizaciones realizadas sirvieron para asegurar el correcto funcionamiento de la herramienta.

- La planificación que realizó la empresa proveedora de software al cliente es fundamental para analizar los avances y resultados en términos de parametrización.
- La capacitaciones que se brindaron al personal antes de la puesta en marcha del software contribuyeron directamente al aprovechamiento y utilización adecuada de la herramienta por parte de los usuarios.

- El software debe ser entregado con manuales y documentos que permitan a los usuarios comprender las tareas y solucionar los problemas que se presenten. De este modo, se evitan las consultas de soporte que pueden demorar la solución de problemas.

- De acuerdo a lo observado, se infiere que un software es una herramienta que permite y mejora la presentación de los procesos para atender una auditoría interna o externa.

- Para la Gerencia de Comercializadora ICER de la Costa mantener los sistemas de gestión a través de un software es beneficioso. Principalmente por la posibilidad de obtener indicadores en tiempo real. Este monitoreo ha permitido a la Gerencia mejorar su toma de decisiones, que es una función vital en dicho cargo.

- El cumplimiento que da el software a los requisitos de la norma técnica NTC ISO 9001:2008 es del 72,5\%; mientras que para la norma técnica NTC OHSAS 18001:2007 es del 94,44\%; este indicador es el resultado de identificar los requisitos que son cumplidos a través de menús o funciones frente a los requerimientos de las normas.

Ahora bien, los resultados obtenidos a partir de los seis cuestionarios en los cuatro cargos de ICER Costa: Gerente, Coordinadora QHS, Líderes de proceso y Trabajadores permitieron conocer la percepción que tienen los usuarios frente al cumplimiento de valor de las promesas del software. Estos resultados se organizaron totalizando las respuestas de los cuestionarios según la aplicación de las siguientes promesas: 
- Facilidad en la instalación del software y puesta a punto

- Capacitación para el manejo de software

- Facilidad de acceso y uso

- Centralización y seguridad de la información

- Contribución al mejoramiento y combinación (integración) de los sistemas

- Disminución de los costos de implementación y mantenimiento

- Facilita la toma de decisiones

- Interfaz intuitiva, amigable y funcional

- Mejora de la comunicación entre procesos

- Mejora la percepción de los usuarios respecto a los sistemas de gestión

- Permite actualizar y consultar los indicadores de los procesos en tiempo real

- Permite la asignación de tareas, notificación y alerta a los usuarios

- Permite mantener, controlar, consultar y administrar la documentación

- Soporte y mantenimiento eficiente y oportuno

Las respuestas afirmativas o negativas se promediaron en cada ítem. Estas se clasificaron siguiendo los colores del semáforo. En este sentido, el color verde significa que las respuestas del ítem fueron en mayor porcentaje afirmativas. El color amarillo significa que existe un porcentaje equitativo entre respuestas afirmativas y negativas, Finalmente, el color rojo significa que el ítem cuenta con un mayor porcentaje de respuestas negativas.

Se encuentran en rojo: a) Facilidad en la instalación del software y puesta a punto, y b) Soporte y mantenimiento eficiente y oportuno. En amarillo se encuentran: a) Contribución al mejoramiento y combinación (integración) de los sistemas, b) Mejora de la comunicación entre procesos, y c) Mejora la percepción de los usuarios respecto a los sistemas de gestión. En verde se encuentran: a) Capacitación para el manejo de software, b) Facilidad de acceso y uso, c) Centralización y seguridad de la información, d) Disminución de los costos de implementación y mantenimiento, e) Facilita la toma de decisiones, f) Interfaz intuitiva, amigable y funcional, g) Permite actualizar y consultar los indicadores de los procesos en tiempo real, h) Permite la asignación de tareas, notificación y alerta a los usuarios, y i) Permite mantener, controlar, consultar y administrar la documentación.

El último vértice de la triangulación se completó a partir de la opinión de los expertos. Dichos datos se obtuvieron a través del envió por correo electrónico de una Matriz de calificación de software que iba acompañada de un informe con los resultados de los vértices anteriores. A través de esta matriz de calificación los expertos evaluaron las características del software ISOSISTEM. Esta matriz buscaba calificar de uno a cinco (siendo cinco excelente y uno malo) las características, estas eran:

La percepción del usuario: 1) Uso de recursos, 2) Facilita la toma de decisiones, 3) Cumplimiento de requisitos normativos, 4) Capacidad para administrar varios sistemas, 5) Reducción de papelería, 6) Reducción de tiempo, 7) Soporte y mantenimiento, 8) Facilidad en el acceso y uso, y 9) Capacitación para uso.

La observación de la investigación con las siguientes 11 características: 1) Exactitud, 2) Adecuación, 3) Rapidez para hacer una tarea, 4) Pruebas antes de uso, 5) Prevención en accesos no autorizados, 6) Protección de la información, 7) Operatividad, 8) Aprendizaje, 9) Atractividad, 10) Facilidad de Instalación, y 11) Adaptabilidad.

La opinión de los expertos frente a ISOSISTEM fue la siguiente: el software ISOSISTEM tuvo un porcentaje de calificación de cinco (5) puntos del 46,03\%, seguido 
de 28,57\% de calificación con cuatro (4) puntos. Es decir, el 74,60\% de la calificación es positiva. Las mejores características que tiene la herramienta son: facilidad en el acceso y uso, así como capacidad para administrar varios sistemas y reducción en papelería. Por otro lado, se puede decir que el soporte y mantenimiento se mantiene como la característica más rechazada.

Por otro lado, los resultados obtenidos a partir de la observación fueron distintos. Estos resultados difieren frente a lo calificado vía correo electrónico por parte de los expertos. En este sentido, frente a la percepción del usuario, los expertos calificaron el software así: el 37,66 \% calificó de cinco (5) puntos el sistema, el 32,47 \% lo calificó con cuatro (4) puntos. Lo anterior quiere decir que el 70,13\% de la calificación fue positiva. Sin embargo, la calificación media y baja fue de 29,87\%. Las mejores características obtenidas a partir de la observación son: Prevención en accesos no autorizados, aprendizaje y atractividad. La adecuación fue la única característica con calificación de cuatro (4) puntos por parte de todos los expertos.

Ahora bien, según los expertos, las características que mayores problemas presenta el software ISOSISTEM son: Protección de la información y exactitud. Características tales como la 'facilidad en la instalación y adaptabilidad' se considera que están en un rango medio de calificación que no ofrece ventajas ni desventajas.

La opinión de expertos frente a otros sistemas de software. Para 'facilidad y practicidad' en el manejo de la información entregada por los expertos el investigador prefirió consolidar las calificaciones de los software mencionados entre los que se encuentra: Isolucion, Alfresco, Docmanager, Share Point y presentar el análisis dentro de la misma línea que el numeral anterior.

De acuerdo a esto, otros software tienen el mismo porcentaje de calificación de cinco (5) puntos del $46,03 \%$ frente a ISOSISTEM. Sin embargo, la siguiente calificación de cuatro (4) puntos es más alta (33,33\%).
Es decir, el 79,37 \% de la calificación es positiva (una cifra mayor que la de ISOSISTEM). Los expertos valoraron las siguientes características como las mejores: Uso de recursos, reducción de papelería y capacidad para administrar varios sistemas. A pesar que los otros software no tuvieron calificación de uno (1), dos expertos calificaron el soporte y mantenimiento con una calificación de dos (2), manteniéndose esta característica como una debilidad característica dentro de los software.

Ahora bien, en términos de la percepción del usuario, los expertos asignaron calificaciones muy parecidas al software ISOSISTEM y al software manejado por ellos: Isolucion, Alfresco, Docmanager y Share Point.

Por otro lado, los expertos confirman que el acceso y facilidad de uso de ISOSISTEM es excelente, sin embargo, mencionan que el soporte y mantenimiento es inadecuado, por lo que consideran que estas últimas deben ser debilidades a corregir. Dentro de la calificación de las características de los otros software los expertos consideran que el uso de recursos y la reducción de papelería es excelente, mientras que el soporte y mantenimiento, la facilidad en el acceso y uso y la capacitación para el uso, obtuvieron una mala calificación.

Frente a la interacción del software con el usuario, los expertos consideran que tanto ISOSISTEM como los otros software tienen valoraciones parecidas. Sin embargo, la calificación más alta de cinco (5) puntos la obtuvieron los otros software con 34 valoraciones frente a 29 del software evaluado.

Para los expertos las características de ISOSISTEM en nivel de excelencia son: la prevención en accesos no autorizados y el aprendizaje (que se refiere a la capacidad de los usuarios para reconocer el concepto lógico y sus aplicaciones). En contraste, las características en nivel deficiente son: la protección de la información. Esta área fue cuestionada, por lo que se considera una debilidad del software. 
En cuanto a los demás software las características que obtuvieron buenas calificaciones fueron: prevención en accesos no autorizados y protección de la información y atractividad. Este último se refiere a las capacidades del software de ser una herramienta visualmente agradable. Por otro lado, las pruebas de uso y la facilidad de instalación fueron duramente cuestionadas.

\section{RESULTADOS A TRAVÉS DE LA VALIDACIÓN DE LA GUÍA DE CALIFICACIÓN DE SOFTWARE}

Teniendo en cuenta las calificaciones asignadas por parte de los expertos, se agruparon las características en sub-características para alinearlas con los requerimientos de la norma técnica ISO/IEC 25000 "System and software quality requirements and evaluation". Además, se definieron las características de acuerdo con los siguientes criterios: si corresponden a requisitos funcionales o requisitos no funcionales.

Las características se clasificaron en la Guía de Calificación de Software según la mayor cantidad de calificaciones que hayan asignado los expertos. En el caso donde dos o más características hayan obtenido diferente calificación, se asigna la más baja. De este modo surgió la Guía de Calificación de Software, la cual ofrece resultados coherentes con la información obtenida de los vértices de la triangulación y de los resultados anteriormente expuestos.

Figura 2. Calificación software ISOSISTEM

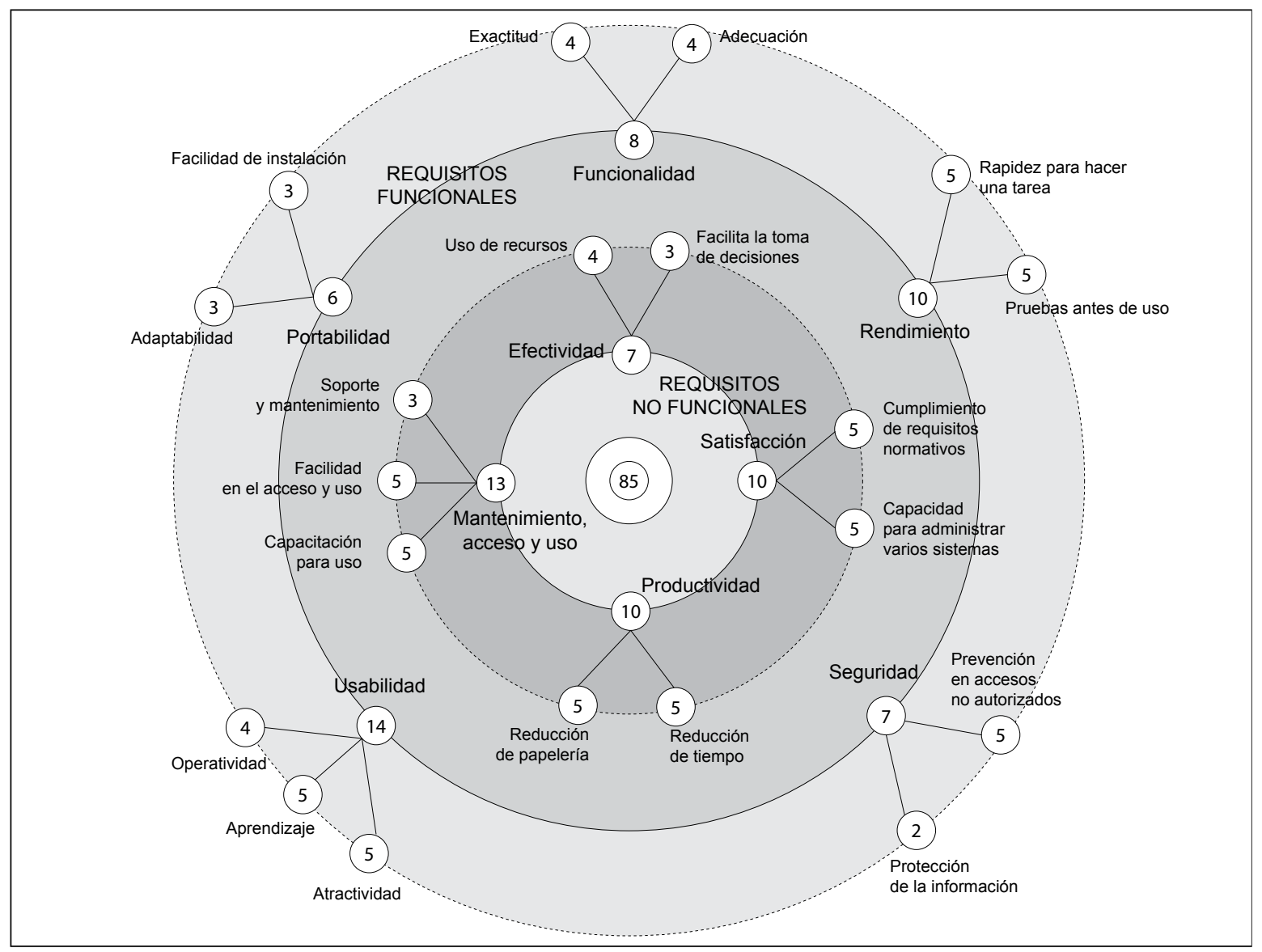

Fuente: elaboración propia. 2014 


\section{CONCLUSIONES}

La comparación de los resultados obtenidos a partir de la observación de la operación del software y de la percepción de los usuarios, junto a la opinión de los siete expertos, permitió la consolidación de la información en atributos que posteriormente fueron definidos como características y subcaracteristicas del software.

Siguiendo el objetivo de determinar la viabilidad y pertinencia del uso del software para administrar el sistema combinado de gestión, se concluye que en ICER Costa sí fue conveniente la adquisición del software porque contribuyó a disminuir los obstáculos presentes en la administración de los sistemas de gestión. Además, según los usuarios, se disminuyeron los costos de implementación y mantenimiento de los sistemas de gestión en un 66,67\%. También se mejoraron al $100 \%$ aspectos tales como: la obtención de información de los indicadores en tiempo real, la asignación de tareas, notificaciones y alertas, así como el control y administración de la documentación.

La hipótesis inicialmente planteada (si utilizar un software trae beneficios para la administración de un sistema combinado de gestión), se ha podido corroborar a través de la aplicación rigurosa de la metodología, técnicas e instrumentos definidos para esta investigación. Por tanto, podemos concluir que la utilización de un software sí trae beneficios para la empresa. Además, podemos concluir que la Guía de calificación de software aplicada y validada con ISOSISTEM, cumple en 85 puntos sobre 100 con las necesidades y expectativas requeridas para mejorar la administración de un sistema combinado de gestión.

Gracias a la opinión de los expertos y la alineación de la información obtenida con la norma técnica ISO/ IEC 25000, agrupamos las variables utilizadas durante la investigación en características y subcaracteristicas que a su vez pertenecen a los requisitos funcionales y no funcionales del software. Lo anterior permitió establecer y proponer una metodología para evaluar la capacidad de un software para administrar sistemas combinados de gestión que se definió en la Guía de calificación de software.

Una de las mayores fortalezas que debe tener la instalación y puesta en marcha de un software en una compañía es la capacitación que se brinde al personal. En este sentido, según lo observado y lo obtenido a partir de las preguntas formuladas, el $63 \%$ de los trabajadores de la muestra considera estar totalmente de acuerdo frente a aspectos como el entendimiento y comprensión del software gracias a las capacitaciones y las competencias del Ingeniero Programador.

Sin embargo, una de las debilidades que más resaltan del software ISOSISTEM, y en general de otros software, tales como Isolucion, Alfresco, Docmanager y Share Point, es la falta de soporte y mantenimiento que ofrecen. Esta característica obtuvo por parte de los expertos una opinión negativa del 42,86\%. La opinión de los usuarios en términos de eficiencia y oportunidad es aún más baja, con una calificación negativa del $10 \%$, situación que puede cambiar completamente la percepción general que se tiene de un software.

Según los usuarios la promesa del software frente a mejorar la capacidad para tomar decisiones se cumple, aunque se relaciona únicamente con la capacidad para tomar decisiones dentro del alcance de los sistemas de gestión, pues consideran que no facilita la toma de decisiones relacionadas con toda la organización. Esto sucede, principalmente, porque algunos aspectos - como el contable y financiero - no han sido tenidos en cuenta, y por tanto sus métricas no están alineadas al sistema combinado de gestión, ni muchos menos hacen parte de las mediciones que realiza el software.

Ningún software puede asegurar el cumplimiento total de los requisitos. Para el caso de ISOSISTEM 
se observó que de los 40 requisitos definidos para el cumplimiento de la norma NTC-ISO 9001 se da cumplimiento a 29, es decir el 72,50\%. Por el contrario, para la norma NTC-ISO 18001 el cumplimiento es mucho mayor (17 de 18 requisitos definidos $(94,44 \%)$. Sin embargo, el nivel de evolución y actualización de la herramienta permite incluir un mayor número de funciones que permita aumentar esos requisitos.

Respecto a la combinación de los sistemas se definieron 40 requisitos de los cuales se cumplieron tan solo $27(67,50 \%)$. Esta situación también permite concluir que la tendencia del software es generar mayor administración para cada sistema de forma aislada. En este sentido, es en gran medida habilidad del administrador lograr combinar los sistemas de modo que el software ofrezca los resultados deseados.

Aspectos como el manejo de la documentación, registros e indicadores son las mayores ventajas del software que se observaron en procesos de auditoría, pues de las 14 preguntas formuladas $71,43 \%$ tuvieron un impacto positivo. Este resultado surge en gran medida por la consolidación de la información y por el almacenamiento en un solo lugar que facilita y precisa la administración. En este sentido, tener la posibilidad de consolidar la información de los resultados de los indicadores de los procesos y mantenerlos en tiempo real es la mayor ventaja que tiene el software según los usuarios evaluados.

Según los expertos, la calificación del software ISOSISTEM es positiva en un 74,60\% principalmente por la facilidad en su acceso y uso, así como la capacidad para administrar varios sistemas y reducción en papelería. Cabe destacar que se puede contrastar la opinión de los expertos que sí consideran que el software permite administrar varios sistemas con la evaluación del cumplimiento de los requisitos de forma combinada, que alcanzó tan solo un $67,50 \%$ de cumplimiento.
Para esta investigación los resultados finales se dieron en función de la clasificación de las características y subcaracteristicas que hacen parte de los requisitos funcionales y los requisitos no funcionales del software. De esa forma es más fácil calificar la herramienta y determinar qué características o subcaracterísticas se consideran ventajas o desventajas en la utilización del software para administrar un sistema de gestión combinado.

Cada sistema de gestión tiene unas características y particularidades que lo hacen único, pese a que los requisitos normativos son genéricos para cualquier empresa (sin importar su actividad o tamaño). Sin embargo, y de acuerdo a los resultados de la Guía de calificación, el software ISOSISTEM - por su diseño y desarrollo - exige a las empresas y a sus sistemas adaptarse al software. Esta situación se puede dar en parte porque los requisitos funcionales (45/55 puntos) tienen menor cumplimiento que los requisitos no funcionales (40/45 puntos). Es decir que el software tiene mayor cumplimiento en los aspectos externos (no funcionales) que en los aspectos internos (como funciones y menús), los cuales son los elementos que permiten cumplir con las necesidades del sistema combinado de gestión.

\section{RECOMENDACIONES}

Antes de comprar un software para administrar sistemas de gestión es necesario asegurarse de algunos aspectos, tales como:

Soporte y mantenimiento: definir con la empresa proveedora de software un cronograma 0 una agenda de soporte durante y después del montaje del software por un tiempo convenido. Durante este proceso se deben incluir actualizaciones, métodos de soporte y/o mantenimiento, es decir, dejar definidos los momentos en los que la empresa proveedora de software 
pueda dar soporte o mantenimiento. En dicho proceso es también necesario definir mecanismos (ejemplo, asistencia remota, utilizando algún programa que permita al especialista ingresar desde su lugar de trabajo, etc). Así mismo, es necesario definir en qué momento se requiere de la asistencia y presencia física del especialista.

Protección de información: no es suficiente que el software tenga mecanismos de encriptación de la información, es decir, que la información esté correctamente protegida. La máxima protección que puede tener la información que maneja un software se da cuando se tiene un respaldo, es decir, una copia de seguridad (backup) independiente de la infraestructura tecnológica que maneje (ejemplo: servidor, web, 0 en la nube).

\section{REFERENCIAS}

BAUMAN, Zygmunt. La globalización. Consecuencias humanas. En: Sección de obras de sociología. Brasil: Fondo de Cultura Económica. 1999. 23 p.

CERDA. Hugo. Los elementos de la investigación. Bogotá: Editorial El Búho Ltda. 2008. 50 p.

KRISTELL, Ana. Tipos de estudio. Documento en línea: http://www.slideshare.net/kriss2505/tiposde-estudios-3596659

MARTINEZ MIGUELEZ, Miguel. Validez y confiabilidad en la metodología cualitativa. Paradigma [En línea]. 2006, vol.27, n.2, pp. 07-33. ISSN 1011-2251.

ROJAS SORIANO, Raúl. Guía para realizar investigaciones sociales. México: Plaza y Valdez. 1995. 197 p.

YIN, Robert K. Caso de estudio de investigación: diseño y métodos. 3 ed. California: Sage Publicaciones. 1984. 